\title{
Resultate der Umfrage 2017 zur Beurteilung der Weiterbildung
}

\author{
Lea Christina Burgermeister ${ }^{a}$, Bernadette Sütterlin ${ }^{b}, W^{\prime}$ Werner Bauer ${ }^{c}$, Michael Siegrist $^{d}$ \\ a Lic. phil., wissenschaftliche Mitarbeiterin, Consumer Behavior, ETH Zürich; ' ${ }^{b}$ Dr. sc., Senior Researcher, Consumer Behavior, ETH Zürich; \\ ${ }^{c}$ Dr. med., Präsident SIWF; d Prof. Dr. phil., Professor für Consumer Behavior, ETH Zürich
}

\section{Einleitung}

Die Anzahl der Weiterbildungsstellen in der Schweiz basiert nicht primär auf dem Bedarf der Bevölkerung an Fachärzten, sondern viel mehr auf dem Bedarf an Assistenzärztinnen und -ärzten, die für den Klinikbetrieb benötigt werden. Die Frage von Steuerungsmöglichkeiten steht deshalb im Raum.

Bereits heute gibt es Steuerungsmechanismen, durch welche die Anzahl und die Verteilung der Ärztinnen und Ärzte auf die verschiedenen Fachrichtungen und Regionen reguliert werden kann. Dies sind beispielsweise die Erhöhung der Ausbildungsplätze an den medizinischen Fakultäten sowie der Zulassungstopp [1]. Eine Erhöhung der Ausbildungskapazitäten führt jedoch nicht zwangsläufig dazu, dass diese zusätzlichen Ärztinnen und Ärzte sich auch in denjenigen Fachrichtungen spezialisieren, in welchen zusätzlicher Bedarf besteht. Zusätzlich zu den expliziten Steuerungsmassnahmen gibt es weitere Mechanismen, die Einfluss auf die Anzahl und die Verteilung der Ärztinnen und Ärzte in Weiterbildung haben, wie die Einhaltung des Arbeitsgesetzes. Da die Weiterzubildenden zunächst einmal auch Arbeitskräfte sind, welche für den normalen Betriebsablauf benötigt werden, wurde angesichts der limitierten Arbeitszeit vielerorts die Anzahl der Weiterbildungsstellen erhöht. Es ist anzunehmen, dass dieser Ausbau lange nicht immer mit einer tatsächlich erhöhten Kapazität für die Weiterbildung oder einem Bedarf an mehr Fachspezialisten in der Praxis korreliert ist [1].

Die bis jetzt zur Verfügung stehenden Steuerungsmassnahmen setzen mehrheitlich zu Beginn der Ausbildung beziehungsweise nach der Weiterbildung an. Es wäre aber denkbar, durch explizite Steuerungsinterventionen zu versuchen, die Anzahl der Ärztinnen und Ärzte in Weiterbildung auf den zukünftigen Bedarf in den verschiedenen Fachrichtungen auszurichten. Vorstellbar sind finanzielle Anreize oder Vorgaben an die Weiterbildungsstätten, beispielsweise durch eine Festlegung der Anzahl Weiterzubildenden bei der Anerkennung der Weiterbildungsstätten durch das SIWF.
Solche Massnahmen können aber nur dann den gewünschten Effekt haben, wenn der künftige Bedarf an Ärzten in den jeweiligen Fachrichtungen korrekt prognostiziert werden kann.

Heute haben die Ärztinnen und Ärzte in Weiterbildung in der Schweiz sehr grosse Entscheidungsfreiheit bezüglich der Wahl der Fachrichtung. Prinzipiell können sie selbst entscheiden, zu welchem Zeitpunkt sie sich auf eine Fachrichtung festlegen. Die Folge einer allfälligen Umsetzung der einen oder anderen diskutierten Koordinationsmassnahme könnte sein, dass diese Wahlfreiheit der angehenden Fachärztinnen und Fachärzte eingeschränkt wird. Eine frühere Befragung zeigte auf, dass sich etwa die Hälfte der Ärztinnen und Ärzte in Weiterbildung vor Beginn der Weiterbildung für eine Fachrichtung entscheidet [2]. Die andere Hälfte legt sich im Verlauf der Weiterbildung oder danach (2. Facharzttitel) definitiv fest. Im vierten Weiterbildungsjahr steht für einen Grossteil (84\%) der Weiterzubildenden die Entscheidung fest [3]. Für eine wirkungsvolle und sinnvolle Steuerung ist es wichtig, den optimalen Zeitpunkt für die Festlegung auf eine Fachrichtung zu kennen. Deshalb stellt sich die Frage, wann der frühestmöglich zumutbare Zeitpunkt ist.

Das Thema der Steuerung der Anzahl und der Verteilung von Ärztinnen und Ärzten in Weiterbildung sowie die damit zusammenhängende Frage bezüglich des Zeitpunkts der Festlegung für ein Fachgebiet waren Teil der Befragung der Ärztinnen und Ärzte in Weiterbildung und der Leiterinnen und Leiter der Weiterbildungsstätten im Jahr 2017.

\section{Methode}

\section{Umfrageteilnehmer und Durchführung}

An 11521 Ärztinnen und Ärzte in Weiterbildung wurde ein Fragebogen verteilt. Davon haben 8060 diesen retourniert (Rücklaufquote: 70\%). Die Vorgehensweise der Befragung entsprach derjenigen der letzten Jahre [2]. Im Rahmen der Erhebung der statistischen Grund- 


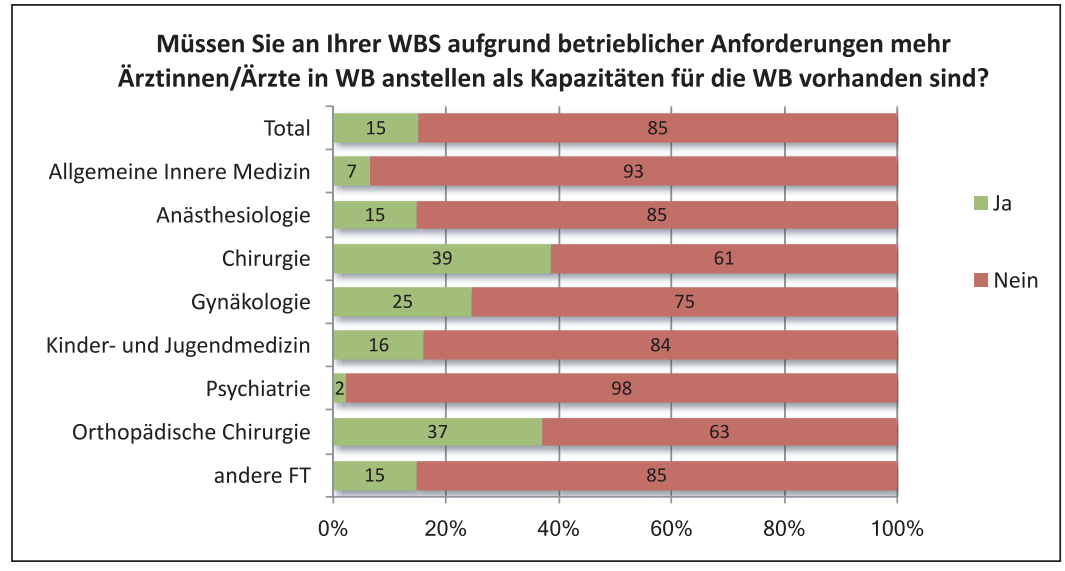

Abbildung 1: Antworten der Leiterinnen und Leiter der Weiterbildungsstätten auf die Frage: "Müssen Sie an Ihrer Weiterbildungsstätte aufgrund betrieblicher Anforderungen mehr Ärztinnen/Ärzte in Weiterbildung anstellen als Kapazitäten vorhanden sind?».

lagen nahmen 1517 Weiterbildungsstättenleiterinnen und -leiter an der Befragung teil (Rücklaufquote: 95\%).

\section{Fragebogen}

Im Fragebogen wurde im variablen Modulteil die Einstellung gegenüber einer gezielten Steuerung der Verteilung und der Anzahl der Weiterbildungsstellen und die Meinung zum Zeitpunkt der Festlegung für eine Fachrichtung erfasst. Die Leiterinnen und Leiter wurden zudem gefragt, ob sie an ihrer Weiterbildungsstätte aufgrund betrieblicher Anforderungen mehr Weiterzubildende anstellen müssen als Kapazitäten vorhanden sind.

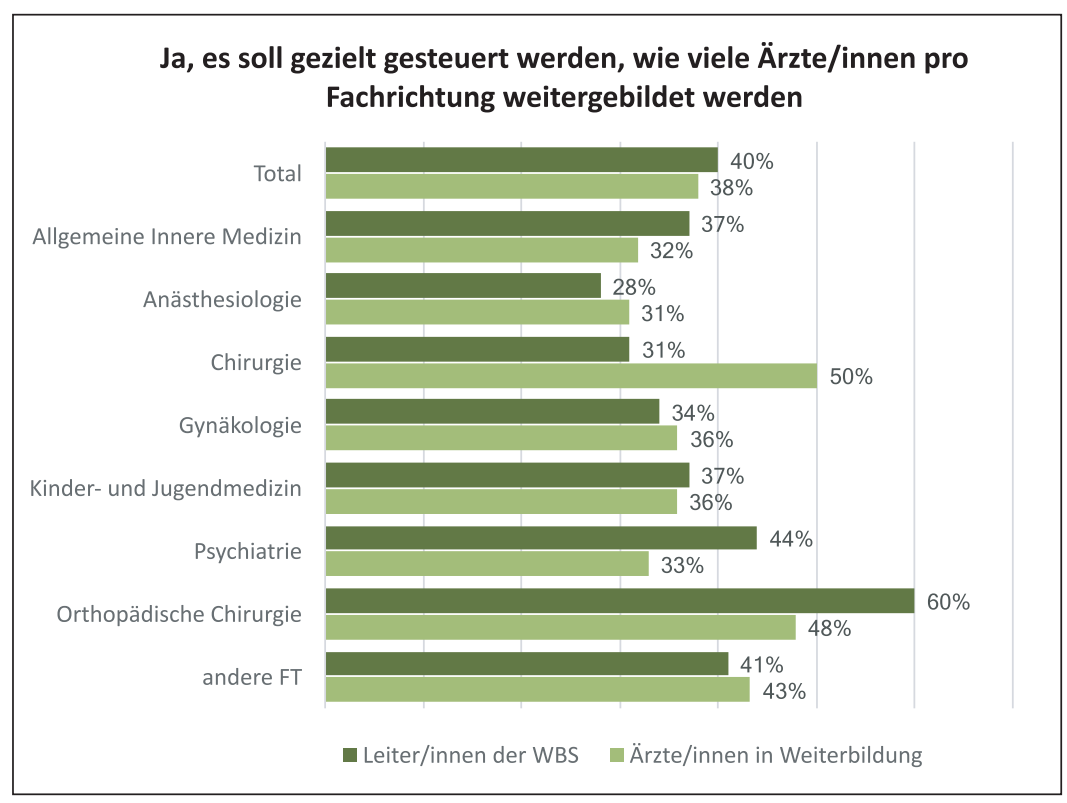

Abbildung 2: Anteil der Ja-Antworten der Leiterinnen und Leiter der Weiterbildungsstätten ( $N=1514$ ) sowie der Ärztinnen und Ärzte in Weiterbildung ( $N=7886$ ) auf die Frage «Soll gezielt gesteuert werden, wie viele Ärztinnen und Ärzte pro Fachrichtung weitergebildet werden (z.B. durch Anreize oder Vorgaben für die WBS)?».

\section{Ergebnisse}

\section{Erhöhung der Weiterbildungsstellen aufgrund betrieblicher Anforderungen}

Von den Leiterinnen und Leitern der Weiterbildungsstätten geben $15 \%$ an, aus betrieblichen Gründen zeitweilig mehr Ärztinnen und Ärzte in Weiterbildung anstellen zu müssen, als Kapazitäten für die Weiterbildung vorhanden sind. Über die häufigsten Fachrichtungen betrachtet, ist der Anteil bei der Chirurgie und der Orthopädischen Chirurgie deutlich höher (Abb.1). In diesen Fachrichtungen machen es betriebliche Gründe heute bei rund einem Drittel der Weiterbildungsstätten hie und da zwingend erforderlich, mehr Weiterzubildende anzustellen, als Kapazitäten vorhanden sind oder als ein gesicherter Weiterbildungsbedarf besteht. Bei der Gynäkologie ist es immerhin ein Viertel der Weiterbildungsstätten.

\section{Gezielte Steuerung}

Von den Leiterinnen und Leitern äusserten sich $52 \%$ gegen eine gezielte Steuerung der Anzahl Ärztinnen und Ärzte pro Fachrichtung, 40\% sprachen sich dafür aus und $8 \%$ waren unentschieden. Bei den Ärztinnen und Ärzten in Weiterbildung liegt die Ablehnung einer gezielten Steuerung etwas tiefer mit 32\%. Die Gruppe der Unentschiedenen ist dafür deutlich höher mit 30\%. Klare Unterschiede zeigen sich in den Sprachregionen. Während in der Deutschschweiz nur gerade ein Drittel (36\%) der Leiterinnen und Leiter zustimmt, findet die gezielte Steuerung in der Romandie und in der italienischsprachigen Schweiz bei der Hälfte Zustimmung (51\% bzw. 46\%).

Deutliche Unterschiede zeigen sich auch zwischen den Fachrichtungen (Abb. 2). Auffallend ist, dass sich in der Orthopädischen Chirurgie eine Mehrheit der Leiterinnen und Leiter (60\%) und der Weiterzubildenden (48\%) für eine Steuerung ausspricht. Der grösste Unterschied zwischen den Leiterinnen und Leitern und den Weiterzubildenden findet sich in der Fachrichtung Chirurgie (31\% vs. $50 \%$ )

Auf deutlich mehr Ablehnung stösst die Idee, bei der Anerkennung einer Weiterbildungsstätte die maximale Anzahl der Weiterbildungsstellen durch das SIWF festlegen zu lassen. Dies lehnen $82 \%$ der Leiterinnen und Leiter $\mathrm{ab}, 10 \%$ sind dafür und $8 \%$ sind unentschieden. In der italienischsprachigen Schweiz liegt die Zustimmung etwas höher (23\%) als in der Deutschschweiz (9\%) und der Romandie (12\%).

Weniger eindeutig präsentiert sich das Bild bei den Ärztinnen und Ärzten in Weiterbildung. Von diesen lehnen 45\% eine Festlegung der maximalen Anzahl 


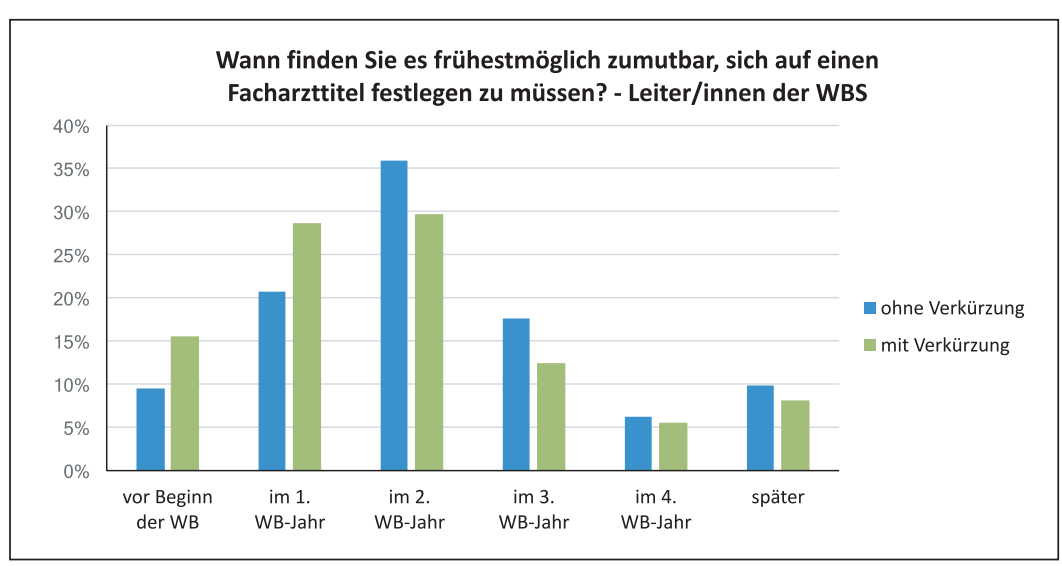

Abbildung 3: Antworten der Leiterinnen und Leiter der Weiterbildungsstätten auf die Fragen: «Wann finden Sie es frühestmöglich zumutbar, sich auf einen Facharzttitel festlegen zu müssen?» und "Wann finden Sie es frühestmöglich zumutbar, sich auf einen Facharzttitel festlegen zu müssen, wenn daraus eine Verkürzung der Weiterbildungsdauer resultiert?».
Bei den Fachrichtungen mit den meisten Weiterzubildenden findet nur bei der Gynäkologie und der Orthopädischen Chirurgie eine Mehrheit der Leiterinnen und Leiter eine Festlegung im ersten Weiterbildungsjahr als zumutbar (Abb. 4). Die Leiterinnen und Leiter der Allgemeinen Inneren Medizin, der Kinder- und Jugendmedizin sowie der Psychiatrie tendieren bezüglich Festlegungszeitpunkt hingegen eher zum dritten Weiterbildungsjahr oder später. Ähnlich sehen dies die Ärztinnen und Ärzte in Weiterbildung.

\section{Diskussion}

Die Resultate der vorliegenden Umfrage zeigen, dass sich die Zustimmung bzw. die Ablehnung der Steuerung der Anzahl und der Verteilung der Ärztinnen und Ärzte in Weiterbildung auf die Fachrichtungen stark zwischen den Sprachregionen und den Fachrichtungen unterscheidet. Während die Leiterinnen und Leiter der Weiterbildungsstätten in der Deutschschweiz klar gegen eine allgemeine gezielte Steuerung sind, stösst diese Massnahme in der Romandie und in der italienischsprachigen Schweiz bei der Hälfte der Leiterinnen und Leiter auf Zustimmung. Dies könnte mit einer, relativ zur Bevölkerung betrachtet, höheren Anzahl an Medizinstudierenden an den Universitäten in der Romandie zusammenhängen. Im Gegensatz zu den anderen Fachrichtungen sind die Weiterzubildenden und die Leiterinnen und Leiter der Orthopädischen Chirurgie sowie die Weiterzubildenden der Chirurgie mehrheitlich für eine Steuerung. Dabei handelt es sich auch um diejenigen Fachrichtungen, bei denen ein Teil der Leiterinnen und Leiter aus betrieblichen Gründen zeitweilig nicht darum herumkommen, mehr Ärztinnen und Ärzte in Weiterbildung anzustellen, als eigentlich Kapazitäten für diese Weiterbildung vorhanden sind.

Deutlicher abgelehnt als die Idee einer allgemeinen Steuerung durch Anreize oder Vorgaben wird die Idee, bei der Anerkennung einer Weiterbildungsstätte die maximale oder totale Anzahl Weiterbildungsstellen durch das SIWF festlegen zu lassen. Dies lehnt die Mehrheit der Leiter und Leiterinnen wie auch der Ärzte und Ärztinnen in Weiterbildung ab.

Die definitive Entscheidung für eine Fachrichtung ist für die meisten Befragten frühestens im zweiten Weiterbildungsjahr zumutbar. In der Psychiatrie, der Allgemeinen Inneren Medizin und der Kinder- und Jugendmedizin tendiert die Meinung eher zum dritten Weiterbildungsjahr oder sogar später. Es ist bemerkenswert, dass genau diese Fachrichtungen (Psychiatrie, Allgemeine Innere Medizin) sich von Versorgungsproblemen bedroht sehen. Möglicherweise wird 


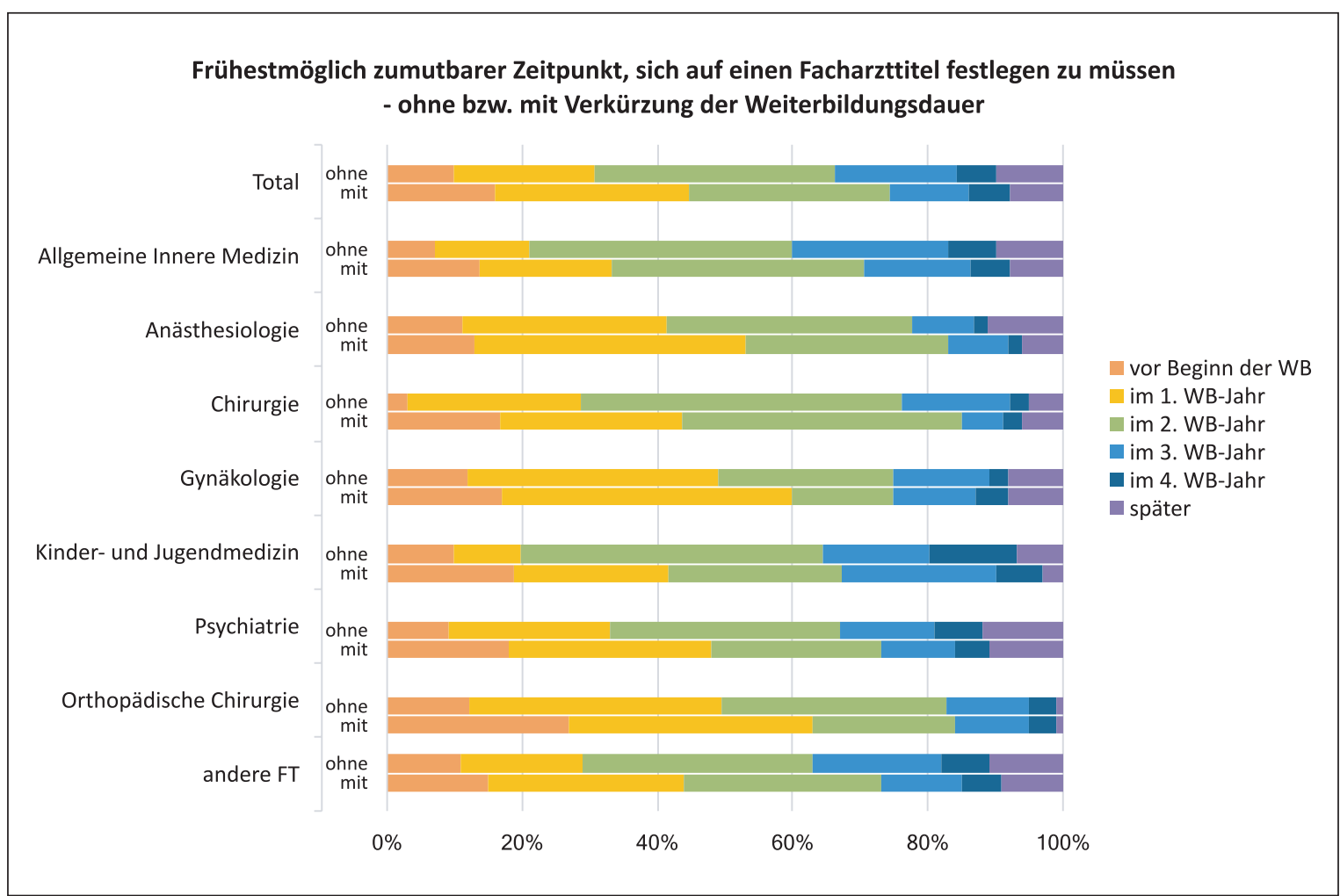

Abbildung 4: Antworten der Leiterinnen und Leiter der Weiterbildungsstätten auf die Fragen: «Wann finden Sie es frühestmöglich zumutbar, sich auf einen Facharzttitel festlegen zu müssen?» (ohne) und «Wann finden Sie es frühestmöglich zumutbar, sich auf einen Facharzttitel festlegen zu müssen, wenn daraus eine Verkürzung der Weiterbildungsdauer resultiert?» (mit).

Korrespondenz: Institute for Environmental Decisions (IED) Consumer Behavior ETH Zürich CHN J 76.3 Universitätstrasse 22 CH-8092 Zürich befürchtet, dass durch eine zu früh angesetzte Entscheidung einige Weiterzubildende davon abgehalten werden könnten, sich für diese Fachrichtungen zu entscheiden. Bei der Kinder- und Jugendmedizin ist es aber so, dass der Entscheid für diese Fachrichtung bei einer grossen Mehrheit bereits vor der Weiterbildung feststeht [2]. Es scheint also nicht grundsätzlich undenkbar, die Wahlfreiheit der Ärztinnen und Ärzte in Weiterbildung etwas einzuschränken und die Entscheidung für eine Fachrichtung auf einen nicht allzu späten Zeitpunkt während der Weiterbildung festzulegen.

Insgesamt zeigt die vorliegende Befragung aber deutlich, dass die Ärzteschaft grosse Vorbehalte gegenüber einer zentralisierten Planung und Steuerung der Anzahl Stellen in den einzelnen Fachrichtungen hat. Weder das BAG noch das SIWF können wirklich zuverlässige Prognosen darüber liefern, in welchen Fachbereichen in zehn bis zwanzig Jahren wie viele Ärzte benötigt werden. Ein Faktor mit entscheidendem Einfluss auf die ärztliche Versorgung der Bevölkerung ist zudem die Immigration, die in alle Überlegungen zur Deckung des Bedarfs einbezogen werden muss, was eine zusätzliche Unsicherheit und Schwierigkeit darstellt. Der «Markt» hat in der Vergangenheit zu einer durchaus brauchbaren Zuteilung geführt, bei der sich nun einige Defizite abzeichnen. Die vorliegenden Ergebnisse zeigen aber eine ausgeprägte Skepsis in der Ärzteschaft gegenüber der Vorstellung, dass eine regulierende Institution so gute Prognosen liefern und so zweckmässige Steuerungsmassnahmen umsetzen kann, dass die Situation wirklich verbessert wird. Eingriffe in die zurzeit ordentlich funktionierenden Mechanismen wären deshalb sehr fragwürdig.

\section{Literatur}

1 Dubach P, Künzi K, Oesch T, Dutoit L, Bannwart L. (2014). Steuerung der ärztlichen Weiterbildung in der Schweiz. Schlussbericht. Bern: Büro für Arbeits- und Sozialpolitische Studien BASS AG.

2 Sütterlin B, Burgermeister LC, Siegrist M, Bauer W. (2016). Resultate der Umfrage 2014 zur Beurteilung der Weiterbildung. Schweiz Ärzteztg, 97(5), 168-71.

3 Buddeberg-Fischer B, Klaghofer R, Abel T, Buddeberg C. (2006). Swiss residents' speciality choices - impact of gender, personality traits, career motivation and life goals. BMC Health Serv Res, 6, 137 